\title{
Fabrication of indocyanine green encapsulated biodegradable microbubbles for structural and functional imaging of cancer
}

\author{
Ronald X. Xu \\ Jiwei Huang \\ Jeff S. Xu \\ The Ohio State University \\ Department of Biomedical Engineering \\ 270 Bevis Hall \\ 1080 Carmack Road \\ Columbus, Ohio 43210 \\ E-mail:xu.202@osu.edu
}

\section{Duxin Sun}

George H. Hinkle

The Ohio State University

Department of Pharmaceutics

251 Parks Hall

500 West 12th Avenue

Columbus, Ohio 43210

\author{
Edward W. Martin \\ Stephen P. Povoski \\ The Ohio State University \\ Department of Surgery \\ Division of Surgical Oncology \\ N-924 Doan Hall \\ 410 West 10th Avenue \\ Columbus, Ohio 43210
}

\begin{abstract}
We developed a novel dual-modal contrast agent for the structural and functional imaging of cancer. The contrast agent was fabricated by encapsulating indocyanine green (ICG) in poly(lacticco-glycolic acid) (PLGA) microbubbles using a modified doubleemulsion method. More stabilized absorption and fluorescence emission characteristics were observed for aqueous and plasma suspensions of ICG-encapsulated microbubbles. The technical feasibility of concurrent structural and functional imaging was demonstrated through a series of benchtop tests in which the aqueous suspension of ICG-encapsulated microbubbles was injected into a transparent tube embedded in an Intralipid phantom at different flow rates and concentrations. Concurrent fluorescence imaging and B-mode ultrasound imaging successfully captured the changes of microbubble flow rate and concentration with high linearity and accuracy. One potential application of the proposed ICG-encapsulated PLGA microbubbles is for the identification and characterization of peritumoral neovasculature for enhanced coregistration between tumor structural and functional boundaries in ultrasound-guided nearinfrared diffuse optical tomography. @ 2009 Society of Photo-Optical Instrumentation Engineers. [DOl: 10.1117/1.3147424]
\end{abstract}

Keywords: microbubble; near infrared; ultrasound; absorption; fluorescence emission; dual-modal imaging.

Paper 08396R received Nov. 10, 2008; revised manuscript received Feb. 23, 2009; accepted for publication Apr. 1, 2009; published online Jun. 8, 2009.

\section{Introduction}

Carcinogenesis represents a process of the development of structural and functional aberrations of normal biological growth patterns at the molecular, cellular, and tissue levels. ${ }^{1,2}$ Simultaneous imaging of tumor structural and functional anomalies is important for accurate detection and appropriate treatment of cancer. However, clinically available hybrid systems, such as SPECT-CT (integrating single photon emission computed tomography and computed tomography) and PET-CT (integrating positron emission tomography and computed tomography), present a radiation hazard and represent significantly added cost, equipment size, and complexity. ${ }^{3}$ Therefore, it is important to develop low cost, portable, nonradiation systems for simultaneous imaging of tissue structural and functional characteristics.

Near-infrared (NIR) imaging is an emerging modality for noninvasive detection of functional anomalies in cancer. ${ }^{2}$ Major advantages of this modality include low cost, portability, deep tissue measurement, molecular sensitivity, and real-time detection of multiple tissue parameters. In this regard, tumor structural and hemodynamic changes in response to external stimuli have been studied for noninvasive detection of tumor

Address all correspondence to Ronald Xu, Biomedical Engineering, The Ohio State University, 270 Bevis Hall-1080 Carmack Rd., Columbus, OH 43210; Tel: 614-688-3635; Fax: 614-292-7301; E-mail: xu.202@osu.edu. dynamic characteristics. ${ }^{4-7}$ Hybrid imaging systems have been developed by integrating NIR with structural imaging modalities, such as ultrasound (US), magnetic resonance imaging (MRI), and mammography. ${ }^{8-11}$ These hybrid systems have used structural imaging modalities to provide spatial guidance for NIR reconstruction of tumor functional properties, with the assumption that tumor structural and functional boundaries are coincident. However, this assumption is not always appropriate. In this regard, a previous comparative study of physical examination, MRI, and mammogram for 20 breast cancer patients following chemotherapy revealed considerable variability in tumor size assessment. ${ }^{12}$ Likewise, a recent, retrospective, finite-element analysis of 10 breast lesions (i.e., five benign and five malignant) suggested that the spatial extent of optical contrast may be significantly larger than the anatomical dimensions reported by structural imaging modalities such as US. ${ }^{13}$ The mismatch between tumor structural and functional boundaries could be caused by inaccurate boundary estimation, inappropriate image coregistration, inflammation, and/or tumor-specific characteristics. Such a boundary mismatch may introduce significant bias when tumor functional properties are reconstructed with a priori guidance of tumor structural boundaries. ${ }^{11,13}$ To overcome this limitation, we fabricated poly(lactic-co-glycolic acid) (PLGA)

1083-3668/2009/14(3)/034020/6/\$25.00 @ 2009 SPIE 
microbubbles encapsulating indocyanine green (ICG) as a dual-modal contrast agent for accurate coregistration between tumor structural and functional boundaries.

ICG is a water-soluble tricarbocyanine dye approved by the Food and Drug Administration (FDA) for many medical imaging applications. ${ }^{14-16}$ In the past, ICG has been used in several cancer imaging and therapeutic applications, including NIR-MRI concurrent imaging of suspicious breast lesions, intraoperative surgical margin assessment and sentinel lymph node mapping and biopsy, ${ }^{17-19}$ and ICG-medicated phototherapy with enhanced cellular uptake and subcellular localization. ${ }^{20}$ However, quantitative analysis of ICG-induced absorption and fluorescence emission is difficult due to its odd optical characteristics contributed by molecular aggregation and protein interaction. In aqueous solution, the absorption and the emission peaks of ICG change with the dye concentration due to molecular aggregation. ${ }^{21}$ In other solutions, such as plasma and human serum albumin, the nonspecific binding of ICG with both lipophilic and hydrophilic molecular species makes its absorption and emission spectra significantly different from those of the aqueous solution. ${ }^{21}$ Furthermore, ICG degrades in aqueous solutions. Degradation is accelerated by light exposure and higher temperature. ${ }^{22} \mathrm{Fi}$ nally, ICG has a short circulation half-life of approximately 2-4 min before it is taken up exclusively by hepatic parenchymal cells and secreted entirely into the bile. ${ }^{21}$ Therefore, in summary, it is very evident that ICG, in its original form, is not ideal for quantitative biomedical imaging applications.

In order to enhance the optical stability of ICG and to extend its circulation lifetime, PLGA nanoparticulate systems entrapping ICG have been developed. ${ }^{23}$ PLGA is an FDAapproved biocompatible and biodegradable material that is widely used in implantation and drug delivery applications. ${ }^{24}$ Gas-filled PLGA microbubbles have been used as a contrast enhancement agent in US imaging. ${ }^{25}$ Recently, the use of microbubbles conjugated with disease-specific ligands has offered clinical US a new opportunity for molecular imaging capabilities. ${ }^{26,27}$ In the arena of optical imaging, microbubbles have been used to enhance the scattering contrast in optical coherence tomography ${ }^{28}$ and diffuse optical spectroscopy. ${ }^{29}$ However, to the best of the present authors' knowledge, microbubbles have not yet been utilized in dual-modal imaging applications. Our current work differs from previous reports in that we are using ICG-loaded microbubbles as a dualmodal contrast agent to coregister between tissue structural and functional anomalies. More stabilized absorption and fluorescence emission characteristics are observed for ICGencapsulated microbubbles in aqueous and plasma solutions due to reduced aggregation and molecular interaction.

\section{Materials and Methods}

\subsection{Materials}

PLGA 50:50 (RG 502H 12000 Da MW) was obtained from Boehringer Ingelheim (Ingelheim, Germany). Polyvinyl alcohol (PVA), methylene chloride $\left(\mathrm{CH}_{2} \mathrm{Cl}_{2}\right)$, and isopropanol $\left(\mathrm{C}_{3} \mathrm{H}_{8} \mathrm{O}\right)$ were purchased from Fisher Scientific (Newton, NJ). ICG was from MP Biomedicals, LLC (Solon, OH). Intralipid-10\%, was obtained from Kabivitrum (Alameda, CA). Ultrapure deionized water was generated by a NAN-

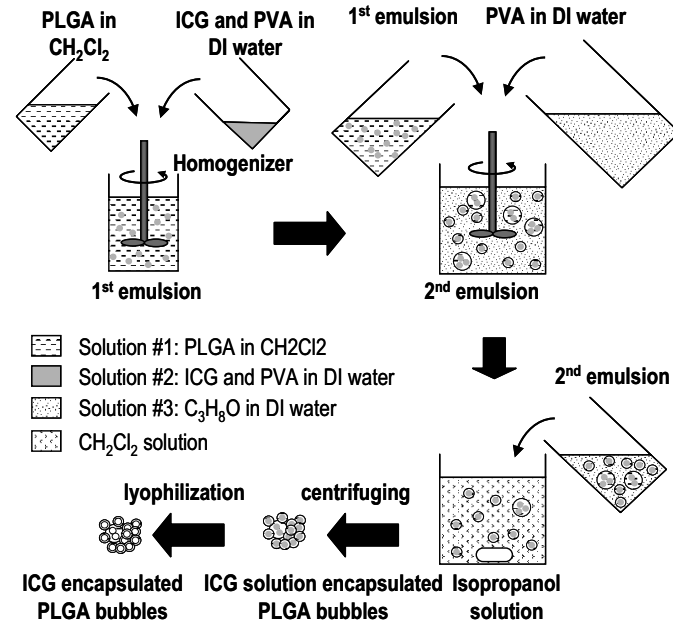

Fig. 1 Fabrication process for ICG-encapsulated PLGA microbubbles.

Opure Infinity water purification system (Barnstead International, Dubuque, IA).

\subsection{Fabrication Process}

ICG-encapsulated PLGA microbubbles were fabricated based on a modified double emulsion process. ${ }^{30}$ The following four solutions were prepared in advance: (i) solution no. 1: $125 \mathrm{mg}$ PLGA in $50 \mathrm{~mL} \mathrm{CH}_{2} \mathrm{Cl}_{2}$, (ii) solution no. 2: $0.65 \mathrm{M}$ ICG and $1 \%$ w/v PVA in $0.5 \mathrm{~mL}$ deionized water), (iii) solution no. 3: $1 \%$ w/v PVA in $50 \mathrm{~mL}$ deionized water, and (iv) solution no. 4: $5 \%$ isopropanol in $100 \mathrm{~mL}$ deionized water. First, solution 2 was added to solution 1 in a $50-\mathrm{mL}$ centrifuge tube and emulsified in an ice bath by a model 17105 Omini mixer homogenizer (Omini International, Waterbury, CT) at 20,000 rpm for $5 \mathrm{~min}$. Then the emulsion was added dropwise using a $1000-\mu \mathrm{L}$ pipette to solution 3 in an ice bath and emulsified by the homogenizer at $500 \mathrm{rpm}$ for $3 \mathrm{~min}$. The double emulsion was then added to solution 4 and stirred for $1.5 \mathrm{~h}$ by a magnetic stirrer in order to extract $\mathrm{CH}_{2} \mathrm{Cl}_{2}$ from the microspheres and harden the PLGA shells. The mixture was then centrifuged by a Centrifuge 5810R (Eppendorf, Hamburg, Germany) at $1500 \mathrm{rpm}$ for $20 \mathrm{~min}$. After centrifugation, the supernatant was discarded and the microbubble precipitate was washed by the deionized water. The process of centrifugation and washing was repeated three times. The washed microspheres were then freeze-dried by a Lyph-lock 4.5 freeze dry system (Labcono Corp., Kansas City, MI) for $36 \mathrm{~h}$. Dried microbubbles were harvested and stored in a glass bottle wrapped with aluminum foil at $0{ }^{\circ} \mathrm{C}$ for further use. The entire fabrication process is illustrated in Fig. 1.

\subsection{Characterization of ICG-Encapsulated PLGA Microbubbles}

A Hitachi S-3000 scanning electron microscope (SEM) (Hitachi High Technologies America, Inc., Pleasanton, CA) was used to image the ICG-encapsulated microbubbles. The size distribution of these microbubbles was characterized by a Dynamic laser scattering system (Particle Sizing Systems, Inc., Santa Barbara, CA). To study the optical characteristics of ICG and ICG-encapsulated microbubbles, the following samples were prepared in $4.3 \mathrm{~mL}$ square cuvettes: $(i) 10 \mu \mathrm{M}$ 


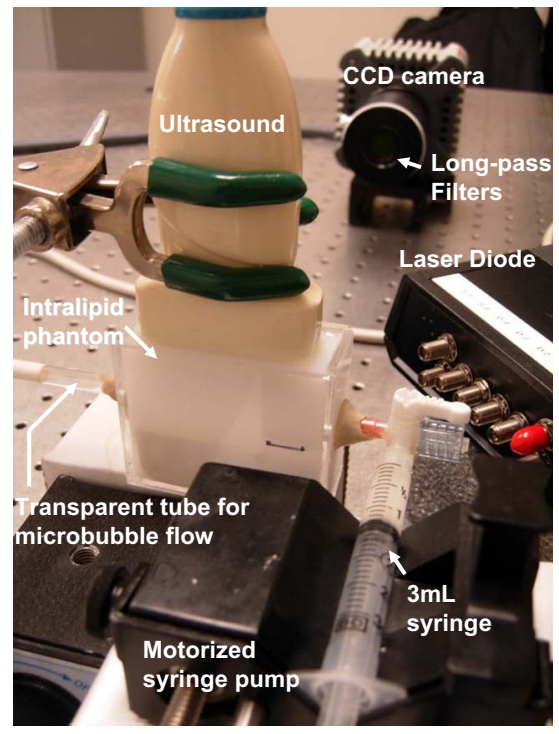

Fig. 2 Benchtop setup for concurrent ultrasound-NIR fluorescence imaging using ICG-encapsulated microbubbles.

ICG in deionized water, (ii) $10 \mu \mathrm{M}$ ICG in bovine plasma, (iii) $10 \mathrm{mg} / \mathrm{mL}$ ICG-encapsulated microbubbles in water, and (iv) $10 \mathrm{mg} / \mathrm{mL}$ ICG-encapsulated microbubbles in bovine plasma. The absorption spectrum of each sample was characterized by a UV-2401PC spectrophotometer (Shimadzu, Columbia, MD). The fluorescence emission of each sample was characterized by an USB4000-FL fluorescence spectrometer (Ocean Optics, Inc., Dunedin, FL) at an excitation wavelength of $690 \mathrm{~nm}$.

\subsection{Concurrent NIR Fluorescence-US Imaging}

As shown in Fig. 2, a benchtop setup was used to demonstrate the technical feasibility of concurrent NIR-US imaging using the ICG-encapsulated microbubble suspension. The benchtop setup consisted of an Intralipid phantom, an ORCA ER deepcooling NIR CCD camera (Hamamatsu, Bridgewater, NJ), FGL715 and FGL780 long pass filters (Thorlabs, Newton, NJ), a Terason 2000 US probe (Teratech Corp., Burlington, MA), and a Qsys2302 laser diode light source (QualSys, Fremont, CA). The phantom was a transparent acrylic box 6.4 $\times 5 \times 2 \mathrm{~cm}$. Diluted Intralipid $10 \%$ solution was filled in the box for a reduced scattering coefficient of $4 \mathrm{~cm}^{-1}$. A transparent plastic tube (radius $2.6 \mathrm{~mm}$ ) was embedded in the diluted Intralipid solution. The center of the tube was placed $5.6 \mathrm{~mm}$ away from the inner wall of the box. The tube had one end open and the other end connected to a 3-mL syringe through a three-way Luer stopcock. The syringe was filled with ICGencapsulated microbubble suspension and was installed on an NE-500 injection pump (New Era Pump System, Inc., Wantagh, NY) for automatic control of flow direction and flow rate. The microbubble solution was excited by the laser diode light source at $690 \mathrm{~nm}$, and the fluorescence emission was captured by the CCD camera with 715- and 780-nm long pass filters. B-mode ultrasound images were simultaneously captured by the US probe.

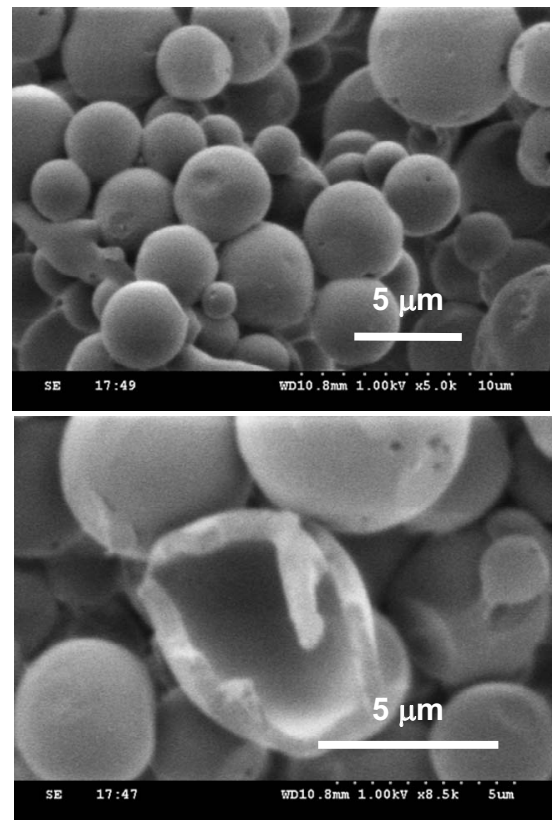

Fig. 3 SEM images of ICG-encapsulated PLGA microbubbles.

\section{Results}

SEM images of ICG-encapsulated PLGA microbubbles are shown in Fig. 3. The cutaway image indicates that the microbubble thickness is around $0.5 \mu \mathrm{m}$. ICG-encapsulated microbubbles have a mean diameter of $3.4 \mu \mathrm{m}$ and a standard deviation of $1.8 \mu \mathrm{m}$. At the current stage of development, we did not strictly control the microbubble size distribution and the ICG encapsulation efficiency. However, these design parameters will provide important control of the US contrast and the NIR contrast in future quantitative imaging applications.

Figure 4 plots the normalized absorption spectra for aqueous solution of ICG, plasma solution of ICG, aqueous suspension of ICG-encapsulated microbubbles, and plasma suspension of ICG-encapsulated microbubbles, respectively. A peak

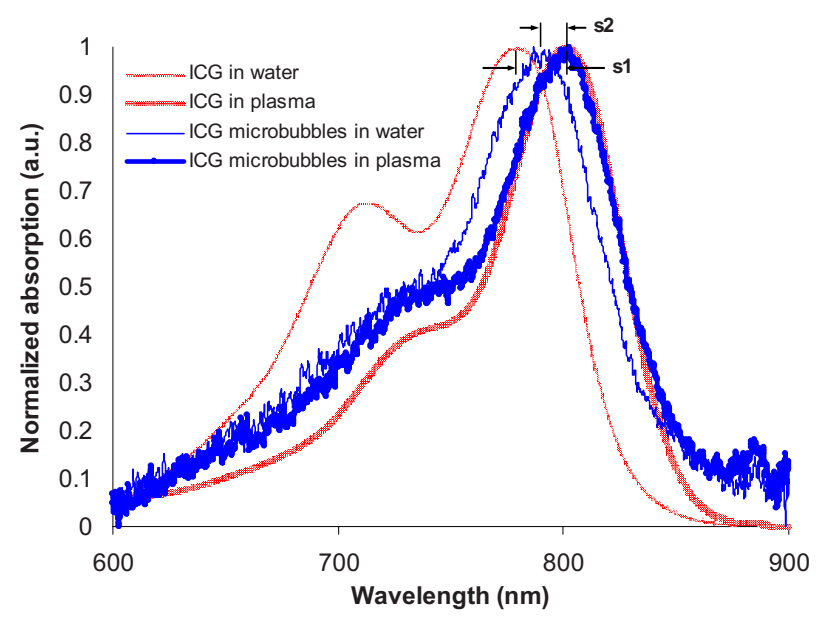

Fig. 4 Normalized absorption spectra for ICG and ICG-encapsulated microbubbles in aqueous and plasma solutions, respectively. Wavelength shift for ICG: $s 1=22 \mathrm{~nm}$; wavelength shift for ICG microbubbles: $\mathrm{s} 2=9 \mathrm{~nm}$. 


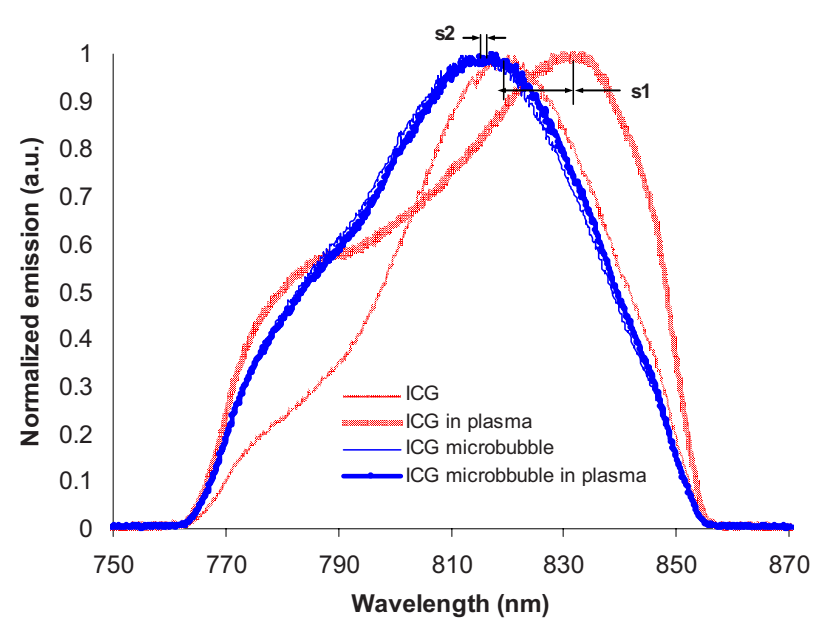

Fig. 5 Normalized fluorescence emission spectra for ICG and ICGencapsulated microbubbles in aqueous and plasma solutions, respectively. S1: $12 \mathrm{~nm}, \mathrm{~S} 2:<1 \mathrm{~nm}$.

wavelength shift of $22 \mathrm{~nm}$ was observed for aqueous and plasma solutions of ICG, whereas such a peak wavelength shift was reduced to $9 \mathrm{~nm}$ for aqueous and plasma suspensions of ICG-encapsulated microbubbles. Figure 5 plots the normalized emission spectra at the excitation wavelength of $690 \mathrm{~nm}$. A peak wavelength shift of $12 \mathrm{~nm}$ was observed in the emission spectra of aqueous and plasma solutions of ICG, whereas the emission shift for aqueous and plasma suspension of ICG-encapsulated microbubbles was less than $1 \mathrm{~nm}$. The reduced wavelength shifts for absorption and emission peaks imply that PLGA encapsulation effectively protects ICG from aggregation and molecular interaction, resulting in more stabilized absorption and fluorescence emission characteristics.

Figure 6 shows typical NIR fluorescence and B-mode US images simultaneously captured by the CCD camera and the US probe as the suspension of ICG-encapsulated mi-

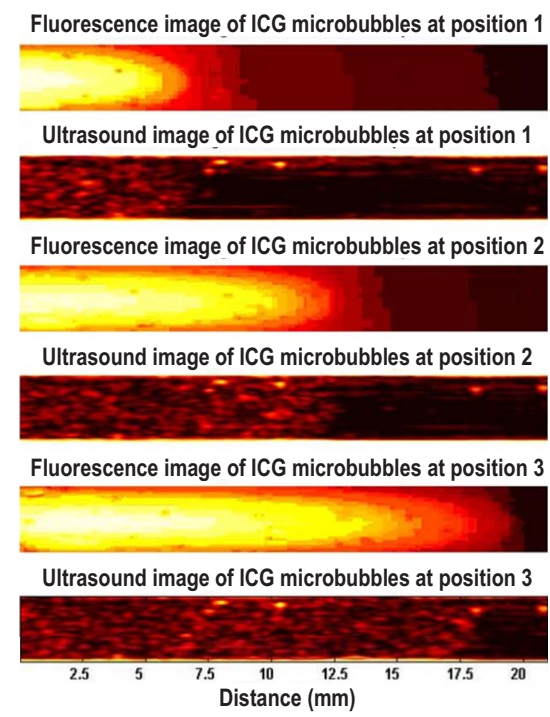

Fig. 6 Typical ultrasound and fluorescence images concurrently captured as ICG-encapsulated microbubbles were injected in a transparent tube. Fluorescence was excited at $690 \mathrm{~nm}$ and imaged after 715 and a 780-nm long pass filters.

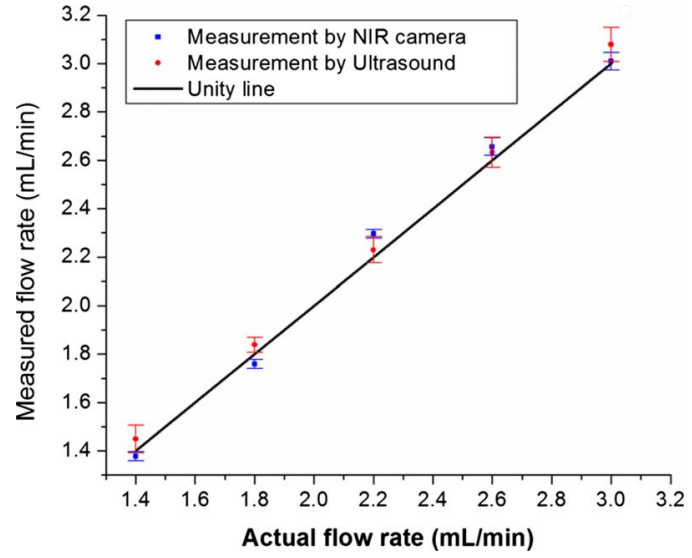

Fig. 7 ICG microbubble flow rates measured from fluorescence images and ultrasound images in comparison with the actual flow rates.

crobubbles was injected in the transparent tube. The images were synchronized with position and time. The test was repeated more than three times with high reproducibility. Front boundaries of the microbubble flow from both NIR fluorescence and US images are coincident, indicating the technical potential for accurate coregistration between structural and functional images using ICG-encapsulated microbubbles. Figure 7 plots the microbubble flow rates calculated from NIR and US images versus the actual flow rates set by the syringe pump. Highly linear correlations $\left(R^{2}>0.9942\right)$ were observed between the measured and the actual flow rates, with a repeatability error of $<4 \%$. Figure 8 plots the normalized fluorescence intensity and the normalized US intensity for various microbubble concentrations ranging from 1.5 to $3.5 \mathrm{mg} / \mathrm{mL}$. Highly linear correlations $\left(R^{2}>0.9803\right)$ were observed between the normalized intensities and the microbubble concentrations, with repeatability error of $<0.8 \%$.

\section{Discussion}

One potential application of the proposed ICG-encapsulated PLGA microbubbles is for the identification and characterization of peritumoral neovasculature for enhanced coregistration between tumor structural and functional boundaries. Hypoxia and angiogenesis are two important characteristics of solid

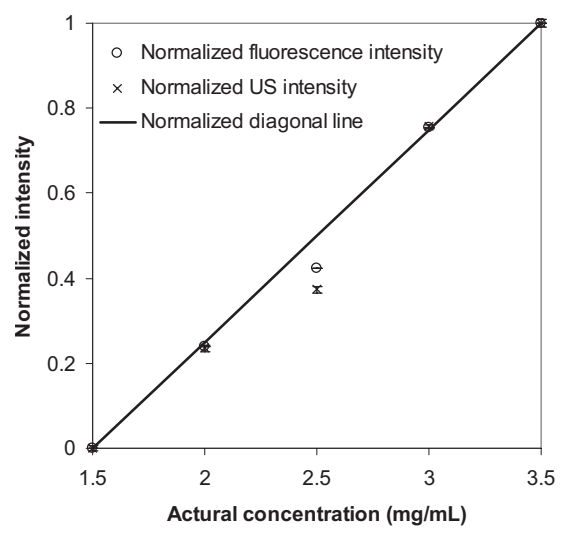

Fig. 8 Normalized fluorescence intensity and ultrasound intensity versus the actual concentration of ICG microbubbles. 


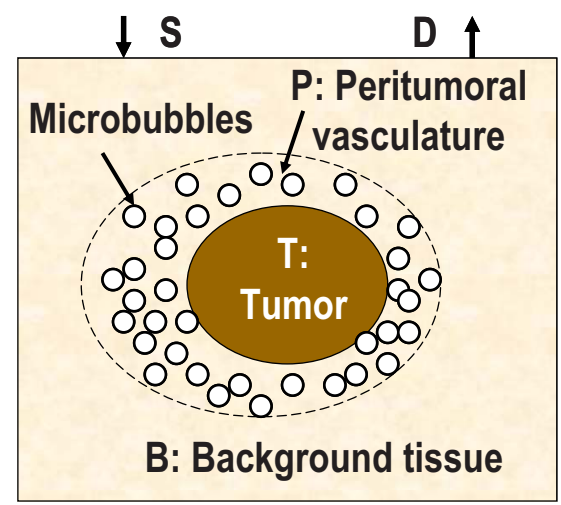

Fig. 9 A simplified model of typical tumor tissue that consists of three compartments: tumor $(T)$, peritumoral neovasculature $(P)$, and background (B).

tumors. $^{31,32}$ Depending on tumor stage and tumor type, a given solid tumor may have a hypoxic or necrotic central core that is surrounded by an extended region of abundant peritumoral neovascularization. While clinical US is able to detect tumor structural heterogeneity, it is less sensitive to the identification and characterization of peritumoral neovasculature. The injection of microbubbles may act to enhance the US contrast for the identification and characterization of peritumoral neovasculature. ${ }^{33}$ Additionally, the optical perturbation induced by ICG-encapsulated microbubbles may help to identify the contribution of peritumoral neovasculature for enhanced reconstruction accuracy in diffuse optical tomography, with the hypothesis that the spatial extent of the optical contrast is greater than that of the US contrast. ${ }^{13}$ For simplicity's sake, the suspicious tissue is divided into three compartments: tumor $(\mathrm{T})$, peritumoral neovasculature $(\mathrm{P})$, and background (B), as shown in Fig. 9. In this figure, "T" refers to the tumor areas without microbubble perfusion, "P" refers to the tumor neovasculature highlighted by microbubbles, "B" refers to the normal surrounding tissue, "S" refers to light sources, and "D" refers to detectors. Numbers of voxels for compartments $\mathrm{T}, \mathrm{P}$, and $\mathrm{B}$ are $p, q$, and $r$, respectively. The number of source-detector pairs is $m$. Born approximation can be used to simulate the scattering part of the photon density $\left[U_{S D}\right]_{m, 1}$ before microbubble injection, ${ }^{34}$

$$
\begin{aligned}
{\left[U_{S D}\right]_{m, 1}=} & {\left[W^{\mathrm{T}}\right]_{m, p}\left[\delta \mu_{a}^{\mathrm{T}}\right]_{p, 1}+\left[W^{\mathrm{P}}\right]_{m, q}\left[\delta \mu_{a}^{\mathrm{P}}\right]_{q, 1} } \\
& +\left[W^{\mathrm{B}}\right]_{m, r}\left[\delta \mu_{a}^{\mathrm{B}}\right]_{r, 1},
\end{aligned}
$$

where $\left\lfloor\delta \mu_{a}^{\mathrm{T}}\right\rfloor_{p, 1},\left\lfloor\delta \mu_{a}^{\mathrm{P}}\right\rfloor_{q, 1}$, and $\left\lfloor\delta \mu_{a}^{\mathrm{B}}\right\rfloor_{r, 1}$ are absorption perturbations of compartments $\mathrm{T}, \mathrm{P}$, and $\mathrm{B}$, respectively. The weight matrix $W$ in expression (1) is calculated from the Green function $G\left(r_{S}, r_{D}\right)$, the baseline measurement $U_{0}$, the voxel volume $h^{3}$, and the optical diffusion coefficient $D$ :

$$
W=G\left(r_{S}, r_{D}\right) U_{0}\left(r_{S}, r_{D}\right) v h^{3} / D
$$

The scattering part of the photon density $\left[U_{S D}^{\prime}\right]_{m, 1}$ after microbubble injection can be expressed as

$$
\begin{aligned}
{\left[U_{S D}^{\prime}\right]_{m, 1}=} & {\left[W^{\mathrm{T}}\right]_{m, p}\left[\delta \mu_{a}^{\mathrm{T}}\right]_{p, 1}+\left[W^{\mathrm{P}}\right]_{m, q}\left[\delta \mu_{a}^{\mathrm{P}}+\delta \mu_{a b}^{\mathrm{P}}\right]_{q, 1} } \\
& +\left[W^{\mathrm{B}}\right]_{m, r}\left[\delta \mu_{a}^{\mathrm{B}}\right]_{r, 1},
\end{aligned}
$$

where $\left\lfloor\delta \mu_{a b}^{\mathrm{P}}\right\rfloor_{q, 1}$ represents the absorption perturbation due to the injection of microbubbles. Subtracting Eq. (1) from Eq. (3) yields

$$
\left[\delta U_{S D}\right]_{m, 1}=\left[U_{S D}^{\prime}\right]_{m, 1}-\left[U_{S D}\right]_{m, 1}=\left[W^{\mathrm{P}}\right]_{m, q}\left[\delta \mu_{a b}^{\mathrm{P}}\right]_{q, 1} .
$$

Because $\left[\delta U_{S D}\right]_{m, 1}$ can be obtained from optical measurements and $\left\lfloor\delta \mu_{a b}^{\mathrm{P}}\right\rfloor_{q, 1}$ can be estimated based on the correlation of optical and US contrasts for the specific concentration of microbubbles, the peritumoral neovasculature weight matrix $\left\lfloor W^{\mathrm{P}}\right\rfloor_{m, q}$ can be inversely calculated. By applying this peritumoral neovasculature weight matrix, it is highly possible to compensate the tumor boundary mismatch for improved reconstruction accuracy in US-guided NIR tomography. Further experiments are necessary for quantitative validation of the proposed strategy.

\section{Conclusions and Future Work}

We have fabricated an ICG-encapsulated PLGA microbubble contrast agent for the structural and functional imaging of cancer. The PLGA encapsulation effectively protected ICG from aggregation and protein interaction, resulting in more stabilized absorption and emission characteristics for quantitative imaging applications. The technical feasibility of concurrent structural and functional imaging was demonstrated through a series of benchtop tests in which NIR fluorescence imaging and US imaging simultaneously captured the flow rates and the concentrations of ICG-encapsulated microbubbles in an Intralipid phantom. One potential application of the proposed ICG-encapsulated PLGA microbubbles is for the identification and characterization of peritumoral neovasculature for enhanced coregistration between tumor structural and functional boundaries in US-guided diffuse optical tomography.

Because ICG and PLGA are both FDA approved biocompatible materials and microbubbles have been used clinically for US contrast enhancement, ICG-encapsulated PLGA microbubbles may introduce fewer clinical safety concerns in comparison with other exogenous contrast agents. To facilitate successful translation from the benchtop to the bedside, the following future works are currently being considered. First, we plan to carry out a quantitative study of microbubble-enhanced diffuse optical tomography using tissue-simulating phantoms with embedded tumor simulators and mismatched structural/functional boundaries. Through this study, we plan to develop and validate the image reconstruction algorithms for ICG-induced absorption and fluorescence perturbations in a turbid medium. We also plan to compare imaging errors with and without NIR-US boundary coregistration. Second, the fabrication process described in the current report has not been optimized for the best performance characteristics of microbubbles. Further improvement and optimization of the fabrication process is necessary for better control of microbubble thickness, size distribution, US sensitivity, and optical contrast. Third, to extend the circulation lifetime in biological systems, we plan to PEGylate the microbubble surface for reduced immunogenicity and protein 
binding. ${ }^{35}$ Thus, we plan to conjugate microbubbles with ligands to target specific biomarkers, such as the increased expression of vascular endothelial growth factor receptors in tumor neovasculature. ${ }^{26}$

\section{Acknowledgments}

This research is sponsored by a DOD Breast Cancer Research Program (Grant No. W81XWH-07). The authors are grateful for the technical and equipment support of Dr. Yi Zhao from the Biomedical Engineering Department, Dr. Robert Lee and Dr. Bo Yu, from the College of Pharmacy, Dr. George Wang from the College of Biological Science, Dr. Jessica Winter from the Chemical and Biomolecular Engineering Department, and Dr. Chandan Sen and Dr. Jay Zweier from the Davis Heart and Lung Research Institute of The Ohio State University.

\section{References}

1. A. Sarasin, "An overview of the mechanisms of mutagenesis and carcinogenesis," Mutat Res. 544(2-3), 99-106 (2003).

2. R. X. Xu and S. P. Povoski, "Diffuse optical imaging and spectroscopy for cancer," Expert Rev. Med. Devices 4(1), 83-95 (2007).

3. P. Zanzonico, L. Dauer, and J. St Germain, "Operational radiation safety for PET-CT, SPECT-CT, and cyclotron facilities," Health Phys. 95(5), 554-570 (2008)

4. B. Wang, S. P. Povoski, X. Cao, D. Sun, and R. X. Xu, "Dynamic schema for near infrared detection of pressure-induced changes in solid tumors," Appl. Opt. 47(16), 3053-3063 (2008).

5. R. X. Xu, B. Qiang, J. J. Mao, and S. P. Povoski, "Development of a handheld near-infrared imager for dynamic characterization of in vivo biological tissue systems," Appl. Opt. 46(30), 7442-7451 (2007).

6. R. X. Xu, D. C. Young, J. J. Mao, and S. P. Povoski, "A prospective pilot clinical trial evaluating the utility of a dynamic near-infrared imaging device for characterizing suspicious breast lesions," Breast Cancer Res. Treat. 9(6), R88 (2007).

7. R. L. Barbour, H. L. Graber, Y. Pei, S. Zhong, and C. H. Schmitz, "Optical tomographic imaging of dynamic features of densescattering media," J. Opt. Soc. Am. A 18(12), 3018-3036 (2001).

8. V. Ntziachristos, A. G. Yodh, M. Schnall, and B. Chance, "Concurrent MRI and diffuse optical tomography of breast after indocyanine green enhancement," Proc. Natl. Acad. Sci. U.S.A. 97(6), 2767-2772 (2000).

9. Q. Zhu, N. G. Chen, D. Piao, P. Guo, and X. Ding, "Design of near-infrared imaging probe with the assistance of ultrasound localization," Appl. Opt. 40(19), 3288-3303 (2001).

10. A. Li, E. L. Miller, M. E. Kilmer, T. J. Brukilacchio, T. Chaves, J. Stott, Q. Zhang, T. Wu, M. Chorlton, R. H. Moore, D. B. Kopans, and D. A. Boas, "Tomographic optical breast imaging guided by threedimensional mammography," Appl. Opt. 42(25), 5181-5190 (2003).

11. R. X. Xu, J. Ewing, H. El-Dahdah, B. Wang, and S. P. Povoski, "Design and benchtop validation of a handheld integrated dynamic breast imaging system for noninvasive characterization of suspicious breast lesions," Technol. Cancer Res. Treat. 7(6), 471-482 (2008).

12. P. T. Weatherall, G. F. Evans, G. J. Metzger, M. H. Saborrian, and A M. Leitch, "MRI vs. histologic measurement of breast cancer following chemotherapy: Comparison with x-ray mammography and palpation," J. Magn. Reson Imaging 13(6), 868-875 (2001).

13. A. Li, J. Liu, W. Tanamai, R. Kwong, A. E. Cerussi, and B. J. Tromberg, "Assessing the spatial extent of breast tumor intrinsic optical contrast using ultrasound and diffuse optical spectroscopy," $J$ Biomed. Opt. 13(3), 30504-30504 (2008).

14. R. C. Benson and H. A. Kues, "Fluorescence properties of indocyanine green as related to angiography," Phys. Med. Biol. 23(1), 159_ 163 (1978).
15. V. V. Kupriyanov, S. Nighswander-Rempel, and B. Xiang, "Mapping regional oxygenation and flow in pig hearts in vivo using nearinfrared spectroscopic imaging," J. Mol. Cell. Cardiol. 37(5), 947957 (2004).

16. A. E. Elsner, Q. Zhou, F. Beck, P. E. Tornambe, S. A. Burns, J. J. Weiter, and A. W. Dreher, "Detecting AMD with multiply scattered light tomography," Int. Ophthalmol. 23(4-6), 245-250 (2001).

17. A. M. De Grand and J. V. Frangioni, "An operational near-infrared fluorescence imaging system prototype for large animal surgery," Technol. Cancer Res. Treat. 2(6), 553-562 (2003).

18. Y. Tajima, K. Yamazaki, Y. Masuda, M. Kato, D. Yasuda, T. Aoki, T. Kato, M. Murakami, M. Miwa, and M. Kusano, "Sentinel node mapping guided by indocyanine green fluorescence imaging in gastric cancer," Ann. Surg. 249(1), 58-62 (2009).

19. M. Fujiwara, T. Mizukami, A. Suzuki, and H. Fukamizu, "Sentinel lymph node detection in skin cancer patients using real-time fluorescence navigation with indocyanine green: preliminary experience," $J$. Plast Reconstr. Aesthet. Surg. (2008).

20. C. Abels, S. Fickweiler, P. Weiderer, W. Baumler, F. Hofstadter, M. Landthaler, and R. M. Szeimies, "Indocyanine green (ICG). and laser irradiation induce photooxidation," Arch. Dermatol. Res. 292(8), 404-411 (2000).

21. T. Desmettre, J. M. Devoisselle, and S. Mordon, "Fluorescence properties and metabolic features of indocyanine green (ICG) as related to angiography," Surv. Ophthalmol. 45(1), 15-27 (2000).

22. V. Saxena, M. Sadoqi, and J. Shao, "Polymeric nanoparticulate delivery system for indocyanine green: biodistribution in healthy mice," Int. J. Pharm. 308(1-2), 200-204 (2006).

23. V. Saxena, M. Sadoqi, and J. Shao, "Indocyanine green-loaded biodegradable nanoparticles: preparation, physicochemical characterization and in vitro release," Int. J. Pharm. 278(2), 293-301 (2004).

24. E. G. de Jalon, M. J. Blanco-Prieto, P. Ygartua, and S. Santoyo, "PLGA microparticles: possible vehicles for topical drug delivery," Int. J. Pharm. 226(1-2), 181-184 (2001).

25. A. C. Fleischer, K. J. Niermann, E. F. Donnelly, T. E. Yankeelov, K. M. Canniff, D. E. Hallahan, and M. E. Rothenberg, "Sonographic depiction of microvessel perfusion: Principles and potential," $J$. $U l$ trasound Med. 23(11), 1499-1506 (2004).

26. F. S. Villanueva, W. R. Wagner, M. A. Vannan, and J. Narula, "Targeted ultrasound imaging using microbubbles," Cardiol. Clin. 22(2), 283-298, vii (2004).

27. J. R. Lindner, J. Song, F. Xu, A. L. Klibanov, K. Singbartl, K. Ley, and S. Kaul, "Noninvasive ultrasound imaging of inflammation using microbubbles targeted to activated leukocytes," Circulation 102(22), 2745-2750 (2000).

28. J. K. Barton, J. B. Hoying, and C. J. Sullivan, "Use of microbubbles as an optical coherence tomography contrast agent," Acad. Radiol. 9 Suppl. 1, S52-55 (2002).

29. Q. Zhu, B. Chance, W. T. Jenkins, and Y. Zhang, "Enhanced optical scattering by microbubbles," Proc. SPIE, 2929, 157-162 (1997).

30. R. A. Jain, "The manufacturing techniques of various drug loaded biodegradable poly(lactide-co-glycolide) (PLGA) devices," Biomaterials 21(23), 2475-2490 (2000).

31. M. Hockel and P. Vaupel, "Tumor hypoxia: Definitions and current clinical, biologic, and molecular aspects," J. Natl. Cancer Inst. 93(4), 266-276 (2001).

32. J. Folkman, "Tumor angiogenesis and tissue factor," Nat. Med. 2(2), 167-168 (1996).

33. S. H. Kook and H. J. Kwag, "Value of contrast-enhanced power Doppler sonography using a microbubble echo-enhancing agent in evaluation of small breast lesions," J. Clin. Ultrasound 31(5), 227238 (2003).

34. M. A. O’Leary, "Imaging with diffuse photon density waves," Ph.D. thesis, University of Pennsylvania (1996).

35. M. Muller, J. Voros, G. Csucs, E. Walter, G. Danuser, H. P. Merkle, N. D. Spencer, and M. Textor, "Surface modification of PLGA microspheres," J. Biomed. Mater. Res. Part A 66(1), 55-61 (2003). 